\title{
Modeling the hardness of the titanium alloy subjected to laser processing
}

\author{
Vladimir Gusev*, Valentin Morozov, and Dmitry Gavrilov \\ Vladimir State University named after Alexander and Nikolay Stoletovs. Russian Federations
}

\begin{abstract}
The article considers the influence of the radiation power $\mathrm{W}$, the longitudinal feed Spr of the laser beam and the distance L from the protective glass of the laser focusing head to the workpiece on the hardness $\mathrm{Hv}$ of the titanium alloy TiTaN. A multi-factor model is established that relates the surface layer hardness to the input factors of laser processing and allows you to quickly assign a laser treatment mode and to control it in order to improve the quality of the processed layer. The power $\mathrm{W}$ has the greatest effect on the hardness of the surface layer. With increasing $\mathrm{W}$, the hardness increases. Increasing the feed Spr leads to a decrease in the $\mathrm{Hv}$ parameter. The effect of the distance $\mathrm{L}$ is similar to the radiation power, but the degree of influence of $\mathrm{L}$ is more than half as small as $\mathrm{W}$. The developed method of operational assignment of the laser processing mode allows to reduce the auxiliary time for performing the technological operation. The research results are relevant for manufacturing enterprises that implement laser processing processes, as well as for design organizations that develop modern laser equipment.
\end{abstract}

\section{Introduction}

Have been studied extensively processing is widely of welding, surface treatment, including surface quenching of metals and alloys [1]. Also have been studied features of structure formation during laser processing [2], surface morphology during selective laser melting of metal powders [3], simulation model of selective laser melting of stainless steel [4]. Features of the free surface of the molten mass, modeling of liquid flow and heat exchange in the melt pool $[5,6]$, physics of complex melt flow and mechanisms of pore formation, splashes [7], influence of surface tension on the morphology of the molten puddle during laser melting, modification of the surface layer of materials, laser-transformational hardening of steel and its alloys [8-10] are determined.The influence of laser heat treatment parameters on the properties of hardened surface layers, including the power of laser radiation to change the hardness of the surface layer of parts, increase the wear resistance of the bearing Assembly by laser deposition [11-13].

The first studies aimed at establishing a multi-factor model of chromium steel hardness after laser treatment were conducted [14]. The physical basis [15] and technological processes of laser processing of materials [16] have been developed, which indicates the creation of a highly efficient resource-saving technology [17].

\footnotetext{
*Corresponding author: prof_gusev@mail.ru
} 
The effect of laser hardening with a round, profiled and oscillating beam on increasing the service life of machine parts is considered [18] and modeling on several tracks of laser hardening of AISI 4140 steel [19].

Laser material processing processes are characterized by a large number of input independent factors and output optimization parameters, that is, they are multi-criteria and multi-parameter.

In this regard, the most effective are not one-factor models, discussed in several of the reviewed literature sources, and the planned multi-factor experimental studies, the implementation of which set of regression equations linking each studied parameter optimization with several factors of the process, providing interactive and a dominant influence on the formation of geometrical and physical-mechanical characteristics of the treated surface layer [20].

In addition, it is important for manufacturing enterprises to know mathematical models that link independent factors of processing processes with the output parameters of the surface layer quality, on the basis of which it is possible to assign reasonable processing modes. In this approach, the development of multi-factor mathematical models of laser processing processes was not given due attention.

The purpose of this research is to establish a multi-factor experimental model, on the basis of which technological elements of the laser treatment mode are quickly assigned to ensure the required hardness of the surface layer of the titanium alloy and optimize the processing modes. The use of the model is relevant for enterprises that develop modern technologies for laser processing of materials, but also for organizations and companies that design new high-performance laser equipment.

\section{Materials and Methods}

Laser treatment of titanium alloy was performed on a multi-beam (42 beams) diode laser complex. Independent factors of laser treatment were selected: radiation power W, longitudinal feed Spr of the laser beam, and distance L from the protective glass of the focusing head to the workpiece surface.

The plan of a multi-factor experiment $\mathrm{N}=2^{\wedge} 3=8$ was implemented. The output parameter of the processing process was represented by the hardness $\mathrm{Hv}$ of surface layer. Samples were processed-washers with a thickness of $7 \mathrm{~mm}$ and a diameter of $30 \mathrm{~mm}$ made of TiTaN alloy. The hardness of the treated surface layer of the parts was measured using a microhard-meter manufactured by CSM. The minimum load on the indenter is $50 \mathrm{mN}$, the maximum load is $30 \mathrm{~N}$, and the loading speed is up to $10 \mathrm{~N} / \mathrm{min}$. The load holding time is unlimited.

\section{Results}

The results of implementation of the stochastic sequence of experiments of the planning matrix are presented in Table 1, the arithmetic Mean values of hardness on the Vickers scale in the right extreme column of Table 1 are obtained from the implementation of three parallel experiments $(n=3)$ for each row of the matrix. 
Table 1. Hardness of the treated surface layer of titanium alloy

\begin{tabular}{|c|c|c|c|c|}
\hline \multirow{2}{*}{ Experiences } & \multicolumn{3}{|c|}{ Independent factors } & Parameter \\
\cline { 2 - 5 } & $\begin{array}{c}\text { Power } \\
\mathrm{W}, \mathrm{kW}\end{array}$ & $\begin{array}{l}\text { Feed } \\
\text { Spr, } \\
\mathrm{mm} / \mathrm{s}\end{array}$ & $\begin{array}{l}\text { Distance } \\
\text { L, mm }\end{array}$ & $\begin{array}{c}\text { Hardness, } \\
\text { GPa }\end{array}$ \\
\cline { 2 - 5 } & $\mathrm{X}_{1}$ & $\mathrm{X}_{2}$ & $\mathrm{X}_{3}$ & $\mathrm{Hv}$ \\
\hline 1 & 5 & 25 & 85 & 426 \\
\hline 2 & 2 & 25 & 85 & 385 \\
\hline 3 & 5 & 10 & 85 & 362 \\
\hline 4 & 2 & 10 & 65 & 301 \\
\hline 5 & 5 & 25 & 60 & 262 \\
\hline 6 & 2 & 25 & 60 & 628 \\
\hline 7 & 5 & 10 & 60 & 247 \\
\hline 8 & 2 & 10 & & \\
\hline
\end{tabular}

Statistical processing of the results of the multi-factor experiment allowed us to obtain the regression equation in the code designation of independent factors in the form:

$$
Y=393.88+45.29 \mathrm{X}_{1}-40.46 \mathrm{X}_{2}+24.38 \mathrm{X}_{3} .
$$

The confidence intervals for the coefficients of the regression equation (1) were $\Delta \mathrm{bi}=$ \pm 10.6 . The interactive effect of the radiation power and longitudinal laser beam feed on the hardness of the surface layer of a titanium alloy is described by the equation:

$$
\mathrm{Hv}=349.2+30.2 * \mathrm{~W}-5.4 * \mathrm{Spr} .
$$

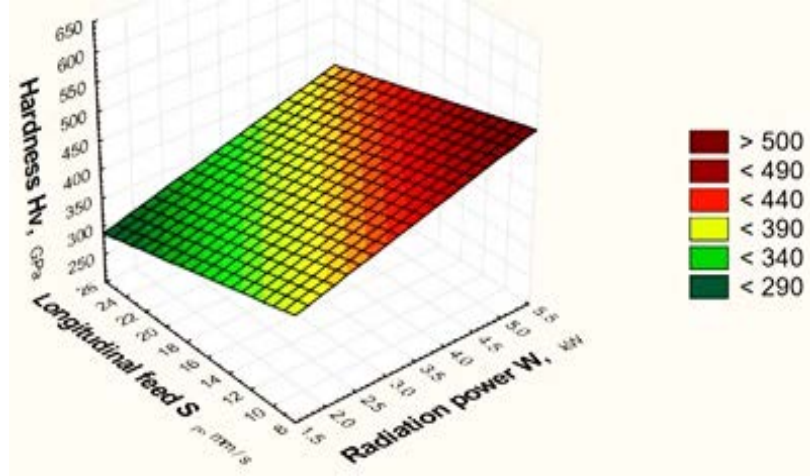

Fig. 1. 3M-XYZ surface-graph of the interactive effect of the radiation power $\mathrm{W}$ and the longitudinal feed Spr of the laser beam on the hardness of the titanium alloy.

From Fig. 1 and equation (2), it can be seen that the laser power $\mathrm{W}$ and the longitudinal feed of the laser beam Spr have a significant effect on the surface layer hardness, while the hardness increases with increasing $\mathrm{W}$ and decreases with increasing Spr. The degree of influence of the laser radiation power is $12.5 \%$ greater than of the longitudinal feed of laser beam. The influence of factors $\mathrm{W}$ and Spr on the hardness is in opposite phase to each other. Contour graphs 3M-XYZ of the interactive influence of independent factors on the optimization parameter are very informative and important for the justification of the laser processing mode. The contour graph $3 \mathrm{M}-\mathrm{XYZ}$ of effect of the radiation power and the longitudinal feed on the hardness of the surface layer is shown in Fig. 2. The interactive effect of power $\mathrm{W}$ and the distance $\mathrm{L}$ on the hardness of the surface layer is described by the regression equation: 


$$
\mathrm{Hv}=146.32+30.17 * \mathrm{~W}+1.96 * \mathrm{~L}
$$

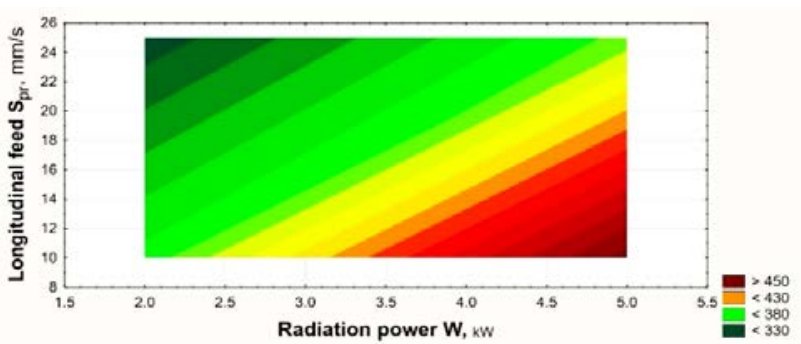

Fig. 2. 3M-XYZ contour graph of the interactive effect of the radiation power $\mathrm{W}$ and the longitudinal feed Spr of the laser beam on the hardness of the titanium alloy.

A graphical interpretation of the multi-factor model (3) of the interactive effect of the radiation power $\mathrm{W}$ and the distance $\mathrm{L}$ from the protective glass to the workpiece surface on the surface layer hardness is shown in Fig. 3 and $3 \mathrm{M}-\mathrm{XYZ}$ contour-graph of this interactive effect is shown in Fig. 4.

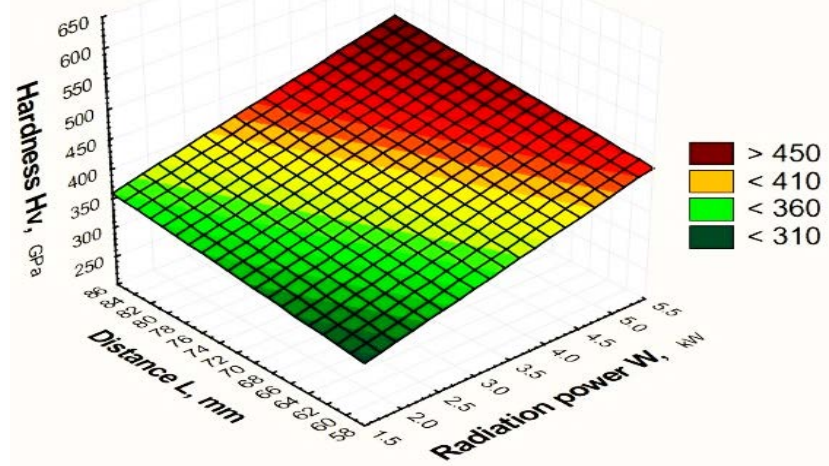

Fig. 3. 3M-XYZ surface-graph of the interactive effect of the power $\mathrm{W}$ and the distance $\mathrm{L}$ on the hardness of the surface layer.

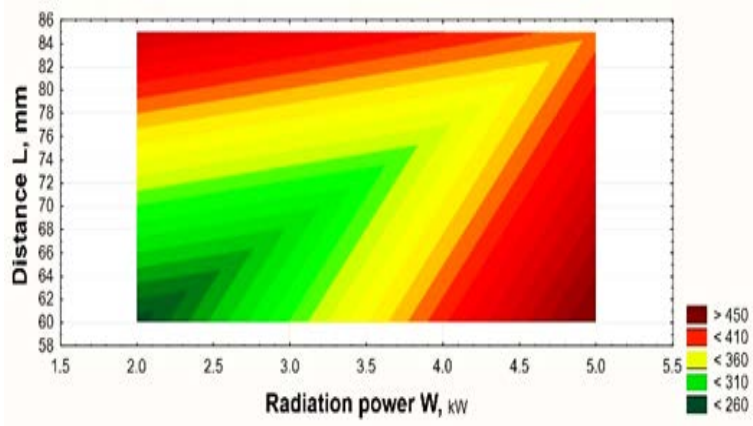

Fig. 4. 3M-XYZ contour-graph of the interactive effect of the laser radiation power $\mathrm{W}$ and the distance $\mathrm{L}$ on the hardness of the titanium alloy.

The interactive effect of the longitudinal feed Spr and the distance L from the protective glass to the workpiece surface on the surface layer hardness is described by the regression equation:

$$
\mathrm{Hv}=312.65-4.05^{*} \mathrm{Spr}+1.96^{*} \mathrm{~L} .
$$


A graphical interpretation of equation (4) is shown in Fig. 5 and 3M-XYZ surface-graph of this interactive effect is shown in Fig. 6.

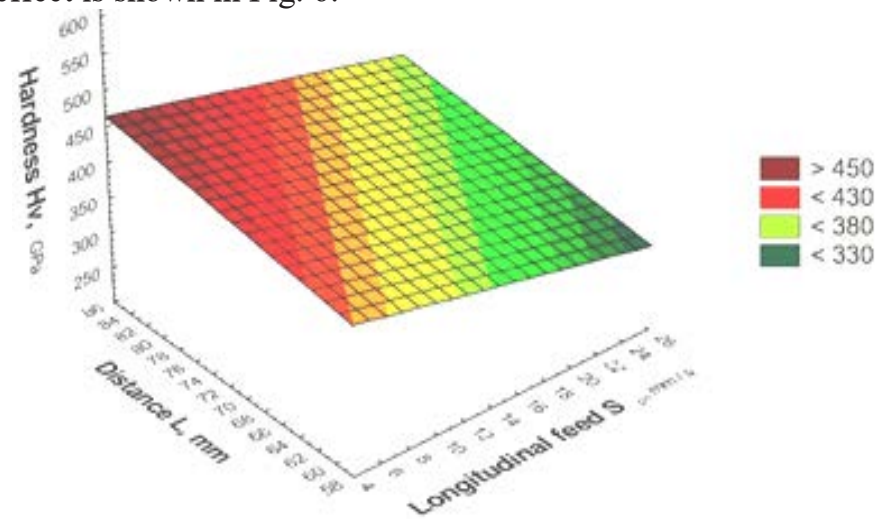

Fig. 5. 3M-XYZ surface-graph of the interactive effect of the longitudinal feed Spr and the distance L on the hardness of a titanium alloy.

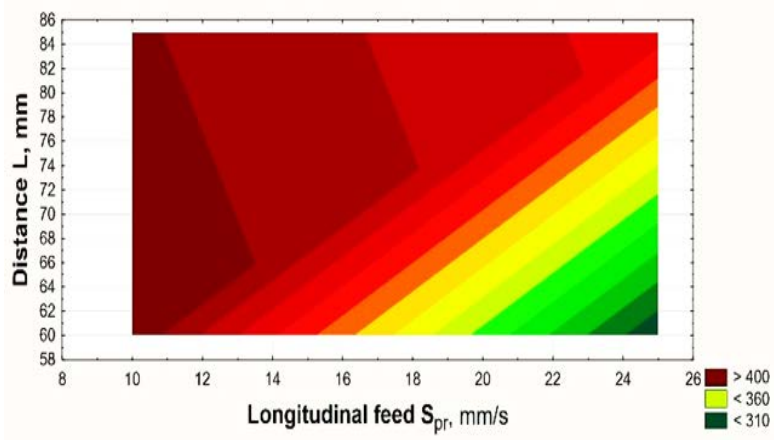

Fig. 6. 3M-XYZ contour-graph of the interactive influence of factors Spr and L on the hardness of the treated surface layer of a titanium alloy.

A multi-factor experimental model that relates the hardness of the treated surface layer to the laser power $\mathrm{W}$, the longitudinal feed Spr, and the distance L from the protective glass to the workpiece surface has the form:

$$
\mathrm{Hv}=86.7+30.17 * \mathrm{~W}-5.4 * \mathrm{Spr}+1.96 * \mathrm{~L}
$$

The regression equations (1)-(4) and their interpretation in the form of graphs (Fig. 1)(Fig. 6) are the basis for the operational assignment of laser treatment modes for titanium alloy, providing the required values of the Hv parameter after processing.

\section{Discussion}

The increase in Hv hardness of the surface layer with an increase in the $\mathrm{W}$ factor is explained by an increase in the thermal load on the surface layer and its rapid cooling by removing heat inside the workpiece material. The decrease in hardness Hv of the surface layer with increasing longitudinal feed of the laser beam can be explained by the reduced time of contact between basic sites of the workpiece with a laser beam, and, therefore, reduced thermal load on the surface layer. Of all the independent factors of the laser processing process the factor $\mathrm{L}$ has the least effect on the hardness of the surface layer. 


\section{Conclusion}

1. An analytical review of scientific works in the field of laser processing of metals and alloys has shown that the main part of the considered works is aimed at identifying the physical nature of phenomena occurring in the zone of laser beam impact on the processed material, and at determining single-factor models that are not suitable for describing multifactor processes.

2. In this regard, developed a multi-factor model of hardness of surface layer of titanium alloy subjected to laser processing that connects the optimization parameter (hardness) with a laser radiation power W, longitudinal feed Spr laser beam and a distance L from the protective glass of the focusing laser head to the workpiece surface.

3. The presented graphical interpretation of the multi-factor model increases the information content of the results obtained and allows you to quickly assign the mode of laser processing of titanium alloy, as well as reduce the time to perform the design procedure.

4. The results of the study are intended for predicting the hardness of titanium alloy and managing processing modes, in order to ensure the required surface layer hardness, and are recommended for use in the design of surface laser processing technology and the development of modern laser equipment.

This work supported by the Ministry of Science and Higher Education of the Russian Federation within framework of the Agreement No 075-15-2019-1838 (project No. 05.607.21.0317, unique ID RFMEFI60719X0317).

\section{References}

1 S.S. Yashkova, J. Young Sci. 1 (135), (2017)

2 N.A. Smirnova, A.I. Misyurov, Bul. of the Bauman Moscow state techn. un-ty, (2012)

3 I. Yadroitsev, I. Smurov, J. Phys. Procedia, 12 (2011)

4 S.A. Khairallah, A.T. Anderson, J. Mater. Process. Technol., 214 (2014)

5 Li Xiang Yang, Xiao Peng Peng, J. Prog. Nat. Sci., 11 (2001)

6 Xiao hu YE, Xi Chen, Chin. J. Lasers, 29 (2002)

7 S.A. Khairallah, A.T. Anderson, A. Rubenchik, at all, J. Acta Mat., 108 (2016)

8 Shibai Liu, Jichang Liu, Jinxuan Chen, at all, Influence of surface tension on the molten pool morphology in laser melting, DOI: 10.1016/j.ijthermalsci.2019.106075.

9 Jan P. Kusinski, Sławomir Kąc, A. Kopia and at all, Review, AGH Un-ty of Sci. and Technol., Poland, (2012)

10 D.B. Purushothaman, M. Ponnusamy, J. Emerging Materials Research, 8 (2), (2019)

11 O.N. Voytovich, I.O. Sokorov, Bul. of the Bel.-Rus un-ty 2 (39), (2013)

12 M.M. Zhuravlev, O.P. Reshetnikova, Rus., Sci. and tech. Bul. of SSTU, 4 (68), (2012)

13 V.I. Shastin, M.I. Ovchinnikova, J. Young Sci. 21 (155), (2017)

14 V. Gusev, V. Morozov, and D. Gavrilovm, EasyChair Preprint 3114, (2020).

15 V.F. Losev, V.Yu. Morozova, and V.P. Tsipilev, Physical bases of laser processing of materials, Rus, Tomsk, (2011)

16 A.G. Grigoryants, I.N. Shiganov, at all, Bul. Laser-inform. 23 (398), (2008)

17 V.I. Yugov, Bul. Laser-inform. 23 (398), (2008)

18 V.P. Biryukov, A.A. Fishkov, D.Yu. Tatarkin, at all, DOI: 10.22184 / 1993-7296.2017.63.3.28.34 tech. equip. and technol.

19 R.S. Lakhkar, Y.S. Shin, J.M. Crane, J. Sci. and eng, A480 (2008)

20 V.G. Gusev, Theory and practice of planning multi-factor experiments, Rus., Vladimir, (2010) 